\title{
Optically Evolved Assembly Formation in Laser Trapping of Polystyrene Nanoparticles at Solution Surface
}

Shun-Fa Wang, ${ }^{\dagger}$ Tetsuhiro Kudo, ${ }^{\dagger}$ Ken-ichi Yuyama,${ }^{*}{ }^{\dagger}$ Teruki Sugiyama, ${ }^{*},{ }^{\dagger, \ddagger}$ and Hiroshi Masuhara*,†

${ }^{\dagger}$ Department of Applied Chemistry and Institute of Molecular Science, National Chiao Tung University, Hsinchu 30010, Taiwan

${ }^{\ddagger}$ Graduate School of Materials Science, Nara Institute of Science and Technology, Ikoma, Nara 630-0192, Japan

SI-1: Video of the transmission imaging during the nanoparticle assembly formation at the $\mathrm{NaCl}$ concentrations of 0 and $100 \mu \mathrm{g} / \mathrm{mL}$.

SI-2: Video of the 1064-nm scattering imaging during the nanoparticle assembly formation at the $\mathrm{NaCl}$ concentrations of 0 and $100 \mu \mathrm{g} / \mathrm{mL}$. 
SI-3: Optical setup for laser trapping experiments

We carried out the trapping experiments using an optical system illustrated in Figure S1. ${ }^{16,17}$ The optical system was constructed on the basis of an inverted microscope (Olympus, IX71) equipped with a confocal scanning system (Olympus, FV300). A linearly polarized laser beam of 1064-nm from $\mathrm{Nd}^{3+}: \mathrm{YVO}_{4}$ laser (Spectra Physics, J20I-BL-106C) was used as a trapping light source and introduced into an objective lens (60 magnification, N.A. 0.90) incorporated in the microscope. The focal volume of the laser in this microscope system was estimated to be about $1 \mu \mathrm{m}^{3}$. The laser power throughout the objective lens was fixed to be $1.4 \mathrm{~W}$. The laser reflected from the sample was blocked with a short pass filter for the observation.

The trapping behavior was observed by transmission/reflection microscopy and reflection microspectroscopy. In transmission microscopy, white light from a halogen lamp (Olympus, U-LH1001R) incorporated in the microscope was introduced into the sample from its above, and transmitted light was detected with a charge-coupled device (CCD) video camera (WATEC, WAT-231S2). In reflection microscopy, white light from a halogen lamp (HOYA-SCHOTT, MegaLight 100) positioned outside the microscope was guided to the objective lens. The sample was illuminated with white light from its bottom, and backwardly scatted light was 
observed with the CCD camera. The color observed here was slightly different from our recent paper, because we used different CCD camera with wavelength dependent sensitivity. ${ }^{16}$ All the transmission, reflection, and 1064-nm scattering images were obtained from one frame of 33 msec width, as the frame number per second of this CCD camera was 30.

In reflection microspectroscopy, the backscattered light was introduced into a confocal pinhole that limits a detection area to a region of $1.5 \mu \mathrm{m}$ in diameter and monitored with a CCD detector (Princeton Instruments, PIXIS 400) coupled with a spectrograph (Princeton Instruments, ACTON SpectraPro 2300i). The detection position was set at the focal spot of the trapping laser by controlling the confocal scanning system. The backscattering spectrum obtained from the sample was calibrated using that measured for an aluminum mirror, namely, the wavelength dependences of incident white light, of the transmittance of optics positioned between the sample and the detector, and of the sensitivity of the detector were corrected. This calibrated spectrum was used as a reflection spectrum, and each one took accumulation time of 0.5 sec. 


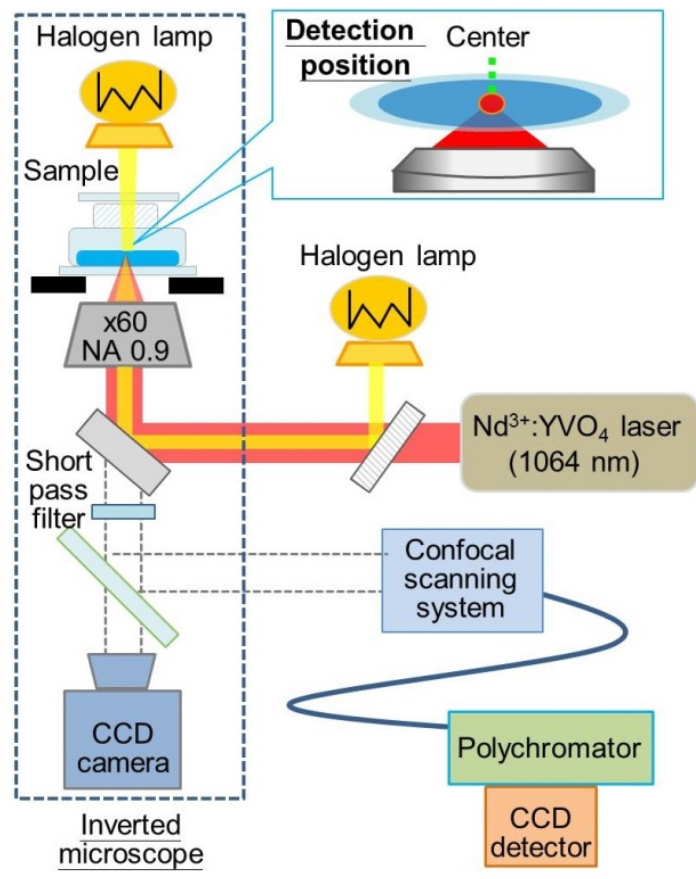

Figure S1. The experimental system for optical trapping, transmission/reflection microscopy, and reflection microspectroscopy. 
SI-4: Transmission images of the single nanoparticle assembly with different numbers

of elongated aggregates

(a)

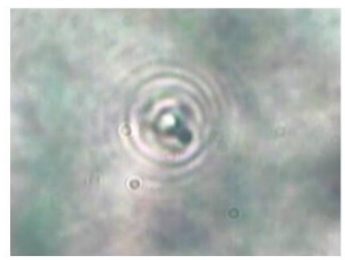

(d)

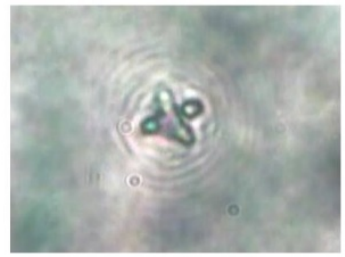

(b)

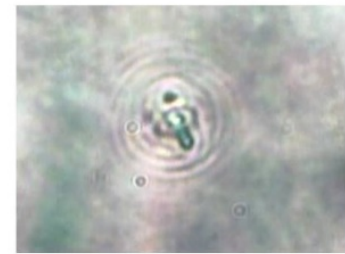

(e)

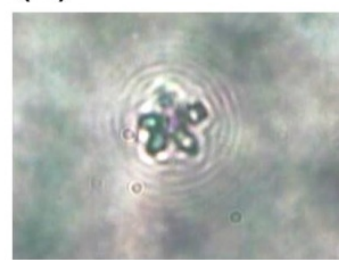

(c)

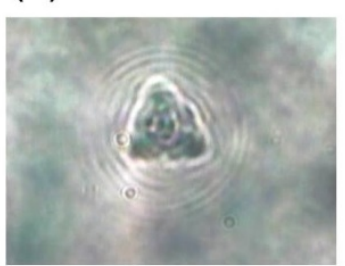

(f)

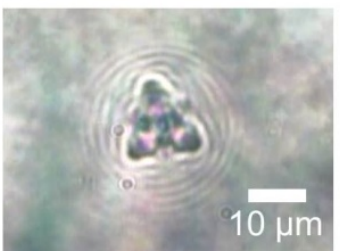

Figure S2. Six examples of the single assembly of nanoparticles containing elongated aggregates observed under the presence of $100 \mathrm{NaCl} \mu \mathrm{g} / \mathrm{mL}$ in the transmission images. 